\title{
Los desafíos de Big Data para los comunicadores y relacionistas colombianos, según datos del LCM 2016-2017
}

\author{
The challenges of Big Data for Colombian communicators and relations, \\ according to data from the LCM 2016-2017
}

\author{
Ana María SUÁREZ MONSALVE ${ }^{1}$ \\ Universidad de Medellín, Colombia \\ asuarez@udem.edu.co
}

Alejandro ÁLVAREZ NOBELL ${ }^{2}$ Universidad Nacional de Córdoba, Argentina aalvareznobell@gmail.com

Recibido:03 de febrero del 2018 Aceptado: 07 de marzo 2018

\section{Resumen}

Este artículo presenta la posición de los profesionales colombianos acerca de Big Data y su uso para la labor comunicacional y de Relaciones Públicas en el país. La segunda edición del Latin American Communication Monitor -LCM 2016-2017, planteó preguntas específicas sobre el conocimiento de Big Data, su uso y proyección de utilización, así como los retos que plantea esta tendencia para el ejercicio profesional. A partir sus respuestas, en este artículo

\footnotetext{
${ }^{1}$ Profesora asociada de la Universidad de Medellín, Colombia. Doctora en Estudios Latinoamericanos de la Universidad de Chile, Magister en Educación de la Pontificia Universidad Javeriana y Comunicadora SocialPeriodista de la Universidad de Antioquia. Asesora en Comunicación Estratégica.

${ }^{2}$ Dircom del Ayuntamiento de Córdoba, Argentina. Doctor en Dirección Estratégica de la Comunicación y Máster en Gestión Estratégica e Innovación en Comunicación de la Universidad de Málaga, España. Licenciado en Comunicación Social de la Universidad Nacional de Córdoba y Licenciado en Periodismo de la Universidad de la Laguna, España.
} 
específica el contexto propio: ¿cuál es el estado de conocimiento, uso y apropiación de Big Data para los profesionales colombianos que participaron en el LCM 2016-2017? Se amplía el análisis con algunos antecedentes sobre el desarrollo de la política pública en Colombia para el uso de datos analíticos y se presentan conclusiones respecto del estado actual de esta tendencia para los profesionales del país.

\section{Summary}

This article presents the position of Colombian professionals about Big Data and its use for communicational work and Public Relations in the country.

The second edition of the Latin American Communication Monitor -LCM 2016-2017 used specific questions about the knowledge of Big Data, its use and usage projection, as well as the challenges posed by this trend for professional practice. Based on your answers, this article specifies your own context: what is the state of knowledge, use and appropriation of Big Data for Colombian professionals who participated in the LCM 2016-2017?

The analysis is broadened with some background on the development of public policy in Colombia for the use of analytical data and conclusions are presented regarding the current state of this trend for professionals in the country.

Palabras clave: Relaciones públicas, Colombia, Big Data, comunicadores

Key Words: Public Relations, Colombia, Big Data, communicators

\section{Introducción}

El Latin American Communication Monitor es un estudio específico sobre la teoría, la práctica y las tendencias de relaciones públicas y comunicación en Latinoamérica, con un antecedente clave de investigación en la región como fue la Macroencuesta Latinoamericana de Comunicación, realizada en 2009 por Ángeles Moreno, Juan Carlos Molleda, Andreia Athaydes y Ana María Suárez Monsalve (. El primer LCM se realizó en 2014-2015, aplicando la valoración de la opinión, de más de 800 casos de profesionales que respondieron la encuesta. En el segundo estudio, LCM 2016-2017, se obtuvo la participación de más de 1000 encuestados y se tomaron 914 casos de profesionales. Esto muestra el aumento en la participación y el interés generado en el estudio.

El LCM 2016-2017 hace parte del Global Communication Monitor que integra los estudios de Europa, Latinoamérica, Asia Pacífico. El objetivo es identificar el desarrollo de la 
comunicación estratégica, conocer su dinámica mediante la comparación en tres segmentos: temas estratégicos, canales de comunicación, desarrollo desde las oportunidades y las necesidades que manifiestan los profesionales en las diferentes regiones. También, la comparación se hace en dos niveles: entre 17 países latinoamericanos y luego, como región, se infieren especificidades relacionadas con las demás regiones del Global Communication Monitor. En la edición 2016-2017 se revisan fenómenos como Big Data, el uso de algoritmos en la función comunicacional y prácticas derivadas de la comunicación como el coaching, a más de indagar por la consultoría y trabajo con agencias.

Los estudios introspectivos de la profesión contribuyen con su institucionalización, el reconocimiento social y la calidad del desempeño de la práctica en el mercado laboral. Así mismo, los estudios longitudinales son análisis científicos que nutren el avance teórico y la investigación aplicada. En un mundo interconectado, con mercados dinámicos y profundamente influyentes en las esferas políticas y económicas de las naciones, el monitoreo de la profesión es vital para su crecimiento con ética e impacto social.

\section{Marco referencial: fundamentación teórica y el contexto del estudio.}

Big Data es entendido como el gran volumen y flujo de diferentes formas de datos procedentes de diversas fuentes (externas e internas) en constante procesamiento, con los que se produce nuevos conocimientos (Gandomi \& Haider, 2015; Schreck et al, 2012, citados en Moreno et al, 2017). La capacidad de almacenamiento, de disponibilidad, inmediatez y de relaciones posibles entre los datos, son condiciones propias del Big Data.

Disponer, de manera fluida y dinámica, de un gran volumen de datos, permite identificar relaciones entre éstos y procesos; generar relaciones inteligentes entre los datos almacenados es la cuestión que interesa. La Revolución Digital ha permitido desarrollar tecnología que reúne más información y la convierte en datos con carácter informativo, de los cuales se extrae valor. Las ciencias de la computación desarrollaron el aprendizaje 
automático para explorar cómo solucionar problemas usando grandes volúmenes de datos almacenados y disponibles, mediante la inteligencia artificial. La premisa es que los macrodatos permiten ver mejor, y de maneras distintas, diversidad de situaciones (Cukier, $\mathrm{K}$, 2017). El valor predictivo de los macrodatos introduce tanto oportunidades como advertencias múltiples.

La Conferencia de Naciones Unidas sobre Comercio y Desarrollo -UNCTAD-, investiga cómo las tecnologías digitales están cambiando la economía y las consecuencias que tiene en el desarrollo. En el Informe sobre la Economía de la Información (2017), advierte que la reducción de costos para almacenar y procesar datos, así como las innovaciones en el procesamiento y digitalización son factores importantes en la transformación de la economía global. La economía digital transforma las cadenas de valor, las necesidades de conocimientos especializados, la producción y el comercio, los marcos jurídicos y normativos en diferentes ámbitos (UNCTAD, 2017, xviii)

La economía digital ha brindado oportunidades como el acceso de pequeñas empresas a mercados de exportación, financiación e información empresarial y, por otro lado, el empoderamiento de la mujer en diferentes ámbitos. Por otro lado, advierte UNCTAD, ha generado la eliminación de algunos tipos de empleo, debido a la automatización de tareas y se cuestiona constantemente, la privacidad de los datos, la seguridad informática y la protección a los derechos de los usuarios (2017, p.4). La cuestión de fondo está en cómo se usan esos datos tanto para las personas individuales como para las empresas y los gobiernos. Como afirma Cukier (2017, p.73), existe otro riesgo y es caer en el dataísmo, es decir, dotar a los datos de más significado de lo que merecen. Por eso, hay que aportar el componente humano de criterio y cordura, es decir, el componente estratégico y ético.

Manuel Castells ha afirmado que el tratamiento de la información en internet constituye una nueva industria, en lo que llama el capitalismo de los datos (2017, p.76), constituida por los sistemas de Big Data que almacena y procesa la información de cada individuo. La 
comunicación digital genera huellas digitales de todos los usuarios, local y globalmente, especialmente a través del uso de las redes sociales.

En el proceso de planificación de este estudio, se encontró que la investigación académica en relaciones públicas y gestión de la comunicación apenas ha comenzado a hacer eco del potencial que tiene Big Data en las funciones y proyecciones de la profesión. Por ejemplo, Big Data facilita el acceso a determinados grupos de stakeholders (Holtzhausen, 2016, citada en Moreno et al, 2017). Permite interpretar el uso de las diferentes plataformas y medios digitales, para reconocer características de los grupos de interés, además de comprender sus comportamientos y tendencias.

Respecto de los algoritmos, entendidos como "un conjunto de reglas que definen con precisión una secuencia de operaciones" (Stone, 1972, citado en Moreno et al, 2017), el registro y medición de los insigths y el engagement de las conversaciones en las redes sociales de una organización, se convierte en una herramienta para escuchar, analizar y guiar la toma de decisiones en comunicación (LCM, 2016-2017, Moreno et al, 2017, p.34).

Tanto Big Data como el uso de algoritmos en redes sociales, han modificado la gestión de la comunicación y han transformado las dinámicas de relacionamiento tanto en el ciberespacio como en las redes analógicas. Las comunidades digitales se organizan e inciden en los escenarios corporativos, trascendiendo las demandas de las audiencias en ciberactivismo. Por tanto, la elaboración de contenidos digitales se ha alimentado con plataformas de inteligencia artificial para hacer difusión de información masiva, instantánea y con datos construidos a través de algoritmos. También los consumidores están cambiando la forma en que se diseñan las campañas publicitarias y de marketing. Los usuarios pagan por bloquear anuncios, por lo que las marcas tienden a concentrarse más en crear contenidos y experiencias, como lo plantea Renato Fernandez, Director Creativo de TBWA/Ciat/Day Los Ángeles (citado en P\&M, 2018, p.40). El viraje hacia la interacción entre las marcas y las audiencias, en vez de mantener la publicidad, allana el camino hacia la generación de 
vínculos y compromiso entre ciudadanos digitales (Robles, 2009) y organizaciones, tanto públicas como privadas.

La revolución digital, la automatización de las relaciones públicas y los nuevos influenciadores en redes sociales, son los temas que más ocupan a los profesionales de la comunicación corporativa y las relaciones públicas en Latinoamérica (LCM 2016-2017).

\section{La comunicación digital en las relaciones públicas y la gestión de comunicación}

Los nuevos líderes de opinión son los influenciadores de las redes sociales (Social Media Influencer-SMI). Estos se han convertido en los defensores de las marcas, por eso, identificarlos y trabajar con ellos se ha vuelto relevante para todo tipo de organizaciones; su importancia radica en el interés de monitorear la reputación y ampliar el alcance de los mensajes dirigidos a las audiencias. Sin embargo, esto requiere una adecuada gestión de datos.

Después de la popularización de los smarthphones, las redes sociales se han incorporado a la vida cotidiana de los ciudadanos y de las organizaciones. La mayor parte de negocios y consumidores se centran en las preocupaciones del día a día y con el uso de sus redes sociales, van marcando preferencias de consumo, temáticas y de actividad social. Otras grandes compañías, se preocupan por identificar los detalles de cada individuo, con las interacciones sociales de cada usuario o consumidor digital.

El Informe Mundial de Tecnología de la Información 2016 del Foro Económico Mundial, afirma que la innovación digital ha sido impulsada principalmente por la demanda de los consumidores. Sin embargo, esta demanda creciente de productos y servicios digitales se satisface por un número relativamente pequeño de empresas (Baller, S, Dutta, S, Lanvin, B., 2016, p.xii). 
El uso de las redes sociales en la gestión de los negocios ha renovado el interés por el seguimiento a los stakeholders. La oferta de plataformas digitales ha facilitado la identificación de los públicos, el análisis de sus necesidades y comportamientos, la creación de contenido para el engagement, incrementando la fidelidad, satisfacción y una percepción positiva de las marcas. Para esto, hay que generar más que interacción, comunicación dialógica, y así construir comunidad digital. Un alto nivel de engagement se relaciona también con el empoderamiento de los ciudadanos digitales.

Entre profesionales de Europa y América Latina que han sido encuestados en ediciones anteriores del LCM, se ha observado una coincidencia de opinión acerca de que no es suficiente la información que ofrecen los servidores de redes para gestionar la comunicación organizacional ni la reputación corporativa. En América Latina ha aumentado la importancia de las redes sociales como canal de comunicación con los públicos, y los profesionales que participaron en el LCM 2014-2015 esperaban una retracción en la importancia de los medios tradicionales por el auge de la comunicación digital. La comunicación y las relaciones públicas estratégicas se muestran abiertas al potencial que brindan las redes sociales, la automatización de la información y Big Data, para construir vínculos fuertes con los públicos y crear comunidades digitales comprometidas con las marcas que promueven el desarrollo, especialmente en países con alta desigualdad social y riesgo económico como Colombia.

\section{Metodología}

Latinamerican Communication Monitor es un estudio longitudinal que se realiza cada dos años, consultando a profesionales de las relaciones públicas, la comunicación estratégica y la gestión comunicacional corporativa en 17 países latinoamericanos: Brasil, Colombia, Argentina, Chile, Perú, Venezuela, Educador, Uruguay, Paraguay, Bolivia, República Dominicana, Costa Rica, Panamá, El Salvador, Guatemala, Honduras, México. Mediante un cuestionario de 33 preguntas que desarrollan 15 secciones temáticas, se analizan 
estadísticamente con SPSS, empleando análisis clúster para clasificar sujetos en algunas de las preguntas que guían la investigación. Se hicieron cruces de variables, con significancia relevante estadísticamente, lograda por la interpretación de coeficientes Chi2, correlación de Pearson, ANOVA/Scheffe Post-hoc, test de independencia de la T de Kendall y el test de correlación de Kendall. El informe completo del LMM 2016-2017 se encuentra disponible en el sitio Web del estudio (www.latincommunicationmonitor.com)

En la edición 2016-2017 se contactaron 2.295 profesionales de 17 países latinoamericanos y completaron el cuestionario 946 profesionales. Por Colombia, se enviaron cerca de 1000 invitaciones y respondieron 160 profesionales, con 142 datos válidos; con base en estos participantes, en este artículo se describen las respuestas para los temas de Big Data y automatización de relaciones públicas, como muestra de la opinión de los relacionistas y comunicadores estratégicos colombianos que participaron en el LCM 2016-2017.

Las características demográficas nos dan una muestra profesionales con cargos directivos ya que un 33,7\% son directores de departamento de comunicación o CEO de agencia, además de un $26,3 \%$ que se identificaron como jefes de equipo o de unidad de comunicaciones y relaciones públicas. El $50 \%$ de los participantes cuenta con 10 años de experiencia en el sector, y la media de edad fue de 39,8 años. El 98\% de los participantes tiene formación universitaria con título propio en el área profesional. Y seis de cada diez profesionales tienen un nivel educativo de máster y doctorado. Esta muestra representa también una distribución del ejercicio laboral en el que poco más de un $80 \%$ están trabajando en empresas (públicas 37 y privadas 45 ) en ONG (18) y 14 trabaja en agencias o como freelance.

Tabla 1. Sector donde trabaja. Muestra de 142 profesionales colombianos participantes del LCM 2016-2017. Fuente: Elaboración propia

\begin{tabular}{|l|l|l|c|}
\hline & Frecuencia & Porcentaje $\begin{array}{c}\text { Porcentaje } \\
\text { válido }\end{array}$ & $\begin{array}{c}\text { Porcentaje } \\
\text { acumulado }\end{array}$ \\
\hline
\end{tabular}




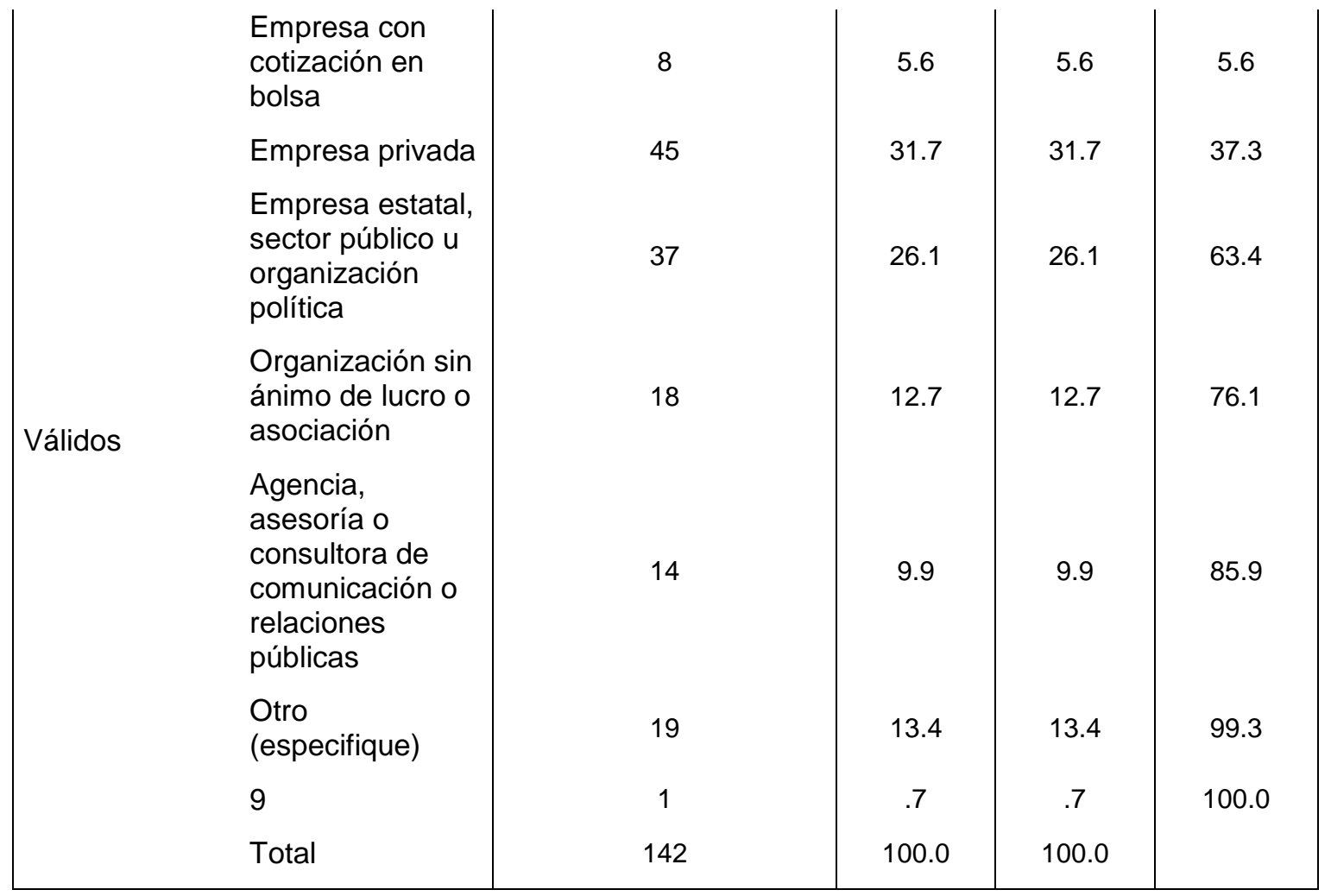

En comparación con la muestra de la región, por los 946 profesionales estudiados en América Latina, Colombia está por debajo en el porcentaje de profesionales que ocupan estos cargos de dirección, ya que, el dato regional en el estudio es de $33,7 \%$ de profesionales directores de departamento o CEO de agencia. Sin embargo, ligeramente está por encima del porcentaje de jefes de equipo de unidad de comunicaciones y relaciones públicas entre los 17 países participantes del LCM 2016-2017.

\section{Resultados}

En primer lugar, se observa que los grandes retos reconocidos por los profesionales, están relacionados con la Evolución Digital y la Web Social (39\%), más Big Data y algoritmos $(37,8 \%)$ como su mayor desafío. Esto, unido al reto constante de identificar, apropiar y 
posicionar el rol de la función de la comunicación (37\%). En ese sentido, se encuentra coherencia entre estos tres desafíos pues la evolución digital ha propiciado la producción de datos en grandes cantidades, de diferente naturaleza, permanentemente y con disponibilidad constante. La disponibilidad de los datos, entonces, no es suficiente; se requiere habilidad técnica, tiempo y análisis para hacer proyecciones constantes, inmediatas y veraces, con esos datos.

Cerca de la mitad de los profesionales consultados (48\%) reconocen que lo han usado y entre estos, un alto porcentaje $(71,2 \%)$ lo orientan a la planificación estratégica. Sin embargo, plantean como desafío mejorar sus habilidades analíticas; en segundo lugar, habilidades técnicas y como dato interesante, el tercer desafío que les presenta el Big Data es la cooperación entre departamentos entre las empresas. El tema de compartir oportunamente la información y la coordinación en la interpretación de los datos. Esto, lo se entiende como un gran desafío para el rol de la comunicación corporativa y las relaciones públicas.

Los profesionales que participaron del LCM 2016-2017, proyectan a 2019 un importante desarrollo de la comunicación digital. Latinoamérica (83\%), así como Asia Pacífico (75\% el porcentaje más alto de los tres canales para los profesionales de Asia Pacífico), muestran una inclinación a considerar que se va a desarrollar más la comunicación con las redes sociales, mientras que los profesionales europeos les dan a estos el porcentaje más bajo (76,2\%). En cambio, la evolución de la comunicación cara a cara es más considerada por los profesionales europeos $(77,6 \%)$ - para Latinoamérica con dos puntos menos $(75,6 \%)$ - pero para Asia Pacífico esa consideración es considerablemente menor $(71,2 \%)$. Es decir, no hay unanimidad al respecto: para los profesionales Latinoamericanos, los canales de comunicación que tendrán mayor relevancia, son las redes sociales y el menor, la comunicación cara a cara. Mientras que, para los profesionales europeos, será la comunicación cara a cara y en menor medida las redes sociales. Y para los profesionales de 
Asia Pacífico, consideran que se desarrollará más la comunicación on line a través de Webs, Email, Intranet y en menor, mucha menor prevalencia, la comunicación cara a cara.

Ante la pregunta sobre si consideran el Big Data como algo importante para la profesión, más profesionales colombianos dijeron estar en total desacuerdo $(22,5 \%)$ y solo en desacuerdo (9,9\%). Adicionalmente, una quinta parte $(21,1 \%)$ no toma partido, no está de acuerdo ni en desacuerdo (Ver Tabla 2). De entrada, estamos ante un grupo de profesionales que no se inscribe en el debate del Big Data ni en sus implicaciones para la profesión, o en las implicaciones para ellos como profesionales de la comunicación y las relaciones públicas. Es menor la representación de profesionales que están de acuerdo $(14,8 \%)$ y totalmente de acuerdo (16,8\%) en que Big Data es importante y tiene implicaciones en el desempeño de las funciones en comunicación estratégica.

Tabla 2. Consideración sobre la importancia de Big Data para los profesionales colombianos participantes del LCM 206-2017. Fuente: Realización propia.

\begin{tabular}{|l|r|}
\hline Es importante Big Data & $\begin{array}{r}\text { Porcentaje } \\
\text { válido } \%\end{array}$ \\
\hline Totalmente en desacuerdo & $22.5 \%$ \\
\hline En desacuerdo & $9 . \%$ \\
\hline Ni de acuerdo ni en desacuerdo & $21.1 \%$ \\
\hline De acuerdo & $14.8 \%$ \\
\hline Totalmente de acuerdo & $16.8 \%$ \\
\hline
\end{tabular}

Con base en estas respuestas, encontramos coherente la afirmación que Moreno et al, 2017, presentan en el informe del LCM 2016-2017, por la cual se percibe un retraso en el desarrollo de esta tendencia, en América Latina. Se desprende esta consideración de datos donde únicamente el $17,6 \%$ de los departamentos y agencias de comunicación 
latinoamericanos informan que han implementado actividades de Big Data. En Colombia, 43,7\% expresa que no lleva a cabo actividades de Big Data (a 2016) en sus actividades profesionales y un $21,8 \%$ ya ha lo ha implementado, el 13,4\% planeaba hacerlo en 2017 y un $16,9 \%$ dice consular a clientes o a otros departamentos y colegas en este campo específico del conocimiento de datos. Es importante señalar que, de quienes sí lo han usado, el 34,8\% dice orientar el uso de Big Data a la planificación estratégica.

En comparación, los profesionales de América Latina que participaron en el estudio muestran que un $52,9 \%$ no ha usado Big Data, pero entre quienes sí lo han usado $(17,6 \%)$, lo hacen para orientar su planificación estratégica $(71,2 \%)$, lo utilizan menos para guiar las acciones del día a día $(58,8 \%)$ y para justificar sus actividades $(53,7 \%)$. Son las empresas que cotizan en bolsa y las organizaciones públicas quienes más lo implementan; quienes trabajan en agencias y consultoras dicen (76,9\%), que no llevan actividades de Big Data.

Esta información es relevante pues, como se puede evidenciar, a 2017, Colombia está un poco retrasada en el uso de Big Data, y mucho más en el uso de esta variedad y volumen de datos para planificar estratégicamente la comunicación y las relaciones públicas. Aun así, Colombia es uno de los países latinoamericanos que muestra uno de los porcentajes más altos en la implementación de Big Data por parte de los profesionales de comunicación $(22,8 \%)$, después de México (24,4\%). Los comunicadores y relacionistas de Argentina $(16,3 \%)$ y Perú $(13,8 \%)$ son los que menos lo han implementado.

Entre las respuestas de los colombianos, también coincide que, quienes usan Big Data, lo hacen siempre para planificar las estrategias generales (siempre $17,6 \%$ y casi siempre 13,4\%), en menor proporción para guiar las acciones del día a día (casi siempre 17,6\%), y como tercera relevancia, para justificar con mediciones sus actividades (casi siempre 12\%)

Tabla 3. Uso de Big Data entre los profesionales colombianos participantes del LCM 2016-2017. Fuente: realización propia.

\begin{tabular}{|l|l|l|}
\hline Uso de Big Data & Colombia & LatAm \\
\hline
\end{tabular}




\begin{tabular}{|l|l|l|}
\hline No lo ha usado & $78.2 \%$ & $52.9 \%$ \\
\hline Orientación a la planificación estratégica & $34.8 \%$ & $71.2 \%$ \\
\hline
\end{tabular}

Tabla 4. Relevancia de los algoritmos en las relaciones públicas para los profesionales colombianos participantes del LCM 2016-2017. Fuente: realización propia.

\begin{tabular}{|l|c|c|}
\hline $\begin{array}{l}\text { ¿Son relevantes los algoritmos y la } \\
\text { tendencia a las relaciones públicas } \\
\text { automatizadas? }\end{array}$ & Uso \\
\hline $\begin{array}{l}\text { Adaptación de algoritmos de } \\
\text { servicios online como motores de } \\
\text { búsqueda }\end{array}$ & $65.9 \%$ & $45.2 \%$ \\
\hline & & \\
\hline $\begin{array}{l}\text { Para la distribución de contenidos: } \\
\text { canales online, listas de correo, } \\
\text { newsletters }\end{array}$ & $\begin{array}{c}\text { Muy } \\
\text { importante }\end{array}$ & Uso \\
\hline & $68.5 \%$ & $52.3 \%$ \\
\hline
\end{tabular}

Al interior de las organizaciones, un reto es la cooperación entre departamentos para tener la información que se requiere oportuna y coherentemente para mayor y mejor análisis corporativo.

Al revisar la distribución del tiempo productivo por parte de los profesionales colombianos participantes del estudio, se encuentra que el mayor número de profesionales (55\%) dedica entre 16 y 30 horas a la semana en labores de gestión de las actividades de comunicación y apoyo a los colaboradores. En comparación de este aspecto, con el resto de comunicadores y relacionistas latinoamericanos, un $27,5 \%$ dedica igual proporción de tiempo a esta función. Esto quiere decir que no es su prioridad productiva, mientras que para los colombianos sí.

El $47 \%$ de los profesionales colombianos, dice que destinan hasta 15 horas, es decir, el $38 \%$ de su tiempo productivo semanal, en hacer orientación, formación y habilitar a los 
colaboradores y directivos. La comparación evidencia que es mayor la destinación de tiempo a esta función por parte de los colombianos, en relación con los profesionales latinoamericanos en total en general $(17,4 \%)$.

A pesar de estas sutiles diferencias, se observa una tendencia similar entre la distribución de tiempo productivo en una semana típica de trabajo de los colombianos y los colegas latinoamericanos. Por ejemplo, se puede observar en la Figura 6, que, en la operatividad de la comunicación se ocupa entre un 34,3\% entre los latinoamericanos y un 34,7\% para los colombianos. Por comunicación operacional se entiende todas las actividades relacionadas con escritura de textos, impresos y digitales, notas de prensa, producción general de medios de comunicación, organización de eventos, monitoreo de procedimientos informativos y comunicativos. En cuanto a la gestión de las actividades de comunicación, es decir, el tiempo dedicado a la planificación, la organización, la evaluación de procesos y estrategias, la realización de presupuestos, prevención de crisis, liderazgo de personal, por ejemplo, se encuentra que, entre los latinoamericanos que respondieron el estudio, destinan un $27,3 \%$ de su tiempo laboral y, los colombianos, dedican un 26,8\%. La armonización de la comunicación, la organización con clientes y stakeholders, es una de las más importantes funciones en cuanto a análisis, toma de decisiones, seguimiento y evaluación, pero, como vemos, tanto latinoamericanos en general $(20,8 \%)$ como colombianos $(20,6 \%)$ destinan a esta función sólo una quinta parte del tiempo productivo.

Tabla 5. Tiempo productivo en una semana de trabajo según los profesionales colombianos participantes del LCM 2016-2017. Fuente: realización propia.

\begin{tabular}{|l|l|l|}
\hline $\begin{array}{l}\text { Tiempo productivo: en una semana típica de trabajo empleo } \\
\text { la siguiente cantidad de tiempo en... }\end{array}$ & LatAm & Colombia \\
\hline Comunicación operacional & $34.3 \%$ & $34.7 \%$ \\
\hline Gestionando actividades de comunicación & $27.3 \%$ & $26.8 \%$ \\
\hline Coaching & $17.4 \%$ & $17.9 \%$ \\
\hline
\end{tabular}

Facultad de Ciencias Sociales y de la Comunicación Universidad de La Laguna 
\begin{tabular}{|l|l|l|l}
\hline Armonizando la comunicación, la organización, clientes y & $20.8 \%$ & $20.6 \%$
\end{tabular} stakeholders

En relación con los retos que impone Big Data y la automatización de relaciones públicas, los profesionales colombianos encuestados requieren, tanto mejorar en sus habilidades analíticas y técnicas, como la destinación de tiempo adecuada para la gestión estratégica y de decisión con los públicos, buscando la armonización de intereses.

\section{Discusión}

En Colombia, el primer paso formalizado para entrar a la era del Big Data, se dio en marzo de 2016 cuando el Departamento de Planeación Nacional presentó el Centro de Excelencia en Big Data y Data Analytics -Alianza Caoba-. Este centro fue cofinanciado por el Ministerio de Tecnologías de la Información y las Comunicaciones (MinTic), en conjunto con Colciencias, con la participación estratégica del sector privado y de universidades, también privadas. Según lo expresó en su momento el Director de Planeación Nacional, Simón Gavira $^{3}$, "Actualmente se toman decisiones con un porcentaje mínimo de los datos e información disponible. Big Data permite acceder y analizar la información a la cual antes no se tenía acceso e, incluso, conocer la opinión de la ciudadanía con precisión, sobre algún tema en particular, en cuestión de horas" (www.dpn.gov.co). Esta premisa de la optimización de los datos, con miras a la democratización y atención de las necesidades del ciudadano, implica tanto una política de datos abiertos u open data, como los datos no estructurados, esto es, los datos que circulan en redes sociales y medios digitales en general. De hecho, el subdirector de TIC del Departamento de Planeación Nacional, Iván Mantilla, planteaba en 2016 que esta iniciativa permitiría analizar y tomar decisiones con respecto a políticas

\footnotetext{
${ }^{3}$ Big Data: Colombia entra en la revolución de los datos. Departamento de Planeación Nacional. Recuperado de https://www.dnp.gov.co/Paginas/Big-Data-Colombia-entra-en-la-revoluci\%C3\%B3n-de-los-datos-.aspx Consultado el 2 de diciembre de 2017.
}

Facultad de Ciencias Sociales y de la Comunicación Universidad de La Laguna 
públicas de manera más eficiente, "Lo que no estamos haciendo en el Estado es coger toda esta 'data', la que está abierta y estructurada y la que no está estructurada, y usarla para comprender las cosas de una mejor manera" (Mantilla en Enter.com, 2016, pp.56-57). La cuestión es clara, desde la definición y aplicación de políticas públicas, Big Data, ofrece la posibilidad de análisis y toma de decisiones, con fines de precisión y optimización de recursos. Sin embargo, el acceso a este volumen y diversidad de información, requiere procedimientos claros y legales, que permitan hacer mejor el trabajo sin violar los derechos de los ciudadanos. Las organizaciones representativas de la comunidad organizada, también están atentas al debate y han advertido los posibles límites que este uso de información puede tener, con miras a proteger los derechos de los ciudadanos, como lo advierte Juan Pablo Castañeda, de la Fundación Karisma, en la revista Enter.co: "el algoritmo de análisis no ha sido suficientemente probado. Se sabe que, en otros países, el resultado de los ejercicios de Big Data ha sido discriminatorio para las personas" (Enter.co. 2016, p. 57). Por esto, está en discusión, tanto en Colombia como en otros países latinoamericanos, el delicado trato de los datos que circulan en el mundo digital y que son soporte de decisiones estatales como, también, en el mundo de la empresa privada. Los profesionales de las relaciones públicas y la comunicación debemos estar atentos a este debate y participar con criterio, sobre los usos adecuados de datos estructurados y no estructurados, para la asesoría estratégica de los clientes y organizaciones que apoyamos.

El uso limitado de Big Data en la región, como, en particular, en Colombia, por parte de los comunicadores y relacionistas, también obedece a la indefinición de políticas que permitan guiar el tratamiento ético de los datos. Además de la falta de habilidades analíticas y técnicas, como se reporta en las respuestas de los profesionales, se suma el poco tiempo que se dedica al análisis estratégico. Se pudo observar, según la opinión de los profesionales colombianos, cómo el mayor tiempo productivo se dedica a la comunicación operacional y a la gestión de la comunicación, y menos tiempo a la alineación estratégica de los públicos. El seguimiento a las interacciones de los diferentes públicos, clientes, 
audiencias y grupos de interés, la interpretación de sus opiniones, la identificación de influencer, la diversidad de opiniones en las distintas redes sobre las organizaciones, la marca, los productos, los servicios, requieren, además de algoritmos de análisis, objetivos claros de seguimiento y procedimientos transparentes ante los públicos. Esto hace que la formación de los profesionales en comunicación y relaciones públicas, incluya tanto la habilidad técnica y analítica, como la orientación filosófica y comunicacional que permita mantener la visión norte del respecto por los públicos, que son, sin duda alguna, ciudadanos globales.

El cuestionario aplicado en esta edición LCM 2016-2017, también consultaba hasta qué punto los comunicadores tenían una comprensión completa del Big Data. Tal como se reporta en el informe (Moreno et al, 2017) un análisis clúster ha permitido clasificar a los encuestados en cuatro grupos: informados, aspirantes, transeúntes y despistados. Los informados $(30,2 \%)$ son quienes prestan atención al debate e información sobre el Big Data y tienen muchos conocimientos en este campo. Por otro lado, el $29,8 \%$ son aspirantes, ya que prestan atención, pero tienen pocos conocimientos sobre Big Data. El resto de los encuestados son transeúntes $(17,2 \%)$, con altos conocimientos y bajo interés, o despistados $(22,8 \%)$, ajenos al debate y el conocimiento en relación con el tema.

En las respuestas de los profesionales se muestra que, evidentemente, son conscientes de la importancia de los algoritmos como motores de búsqueda (6,9\%), para distribución de contenido (61\%), para creación de contenido (55,4\%), para el apoyo en la toma de decisiones $(52,3 \%)$, y para la adaptación de contenido $(41,7 \%)$. Sin embargo, cuando se consultó a los profesionales por la implementación de los algoritmos en algunos de estas formas, el porcentaje no llegó a la mitad. Sólo algunos profesionales informaron que implementan algoritmos para la distribución (49\%) y creación de contenido (39\%) o como motores de búsqueda (39\%). Llama la atención el poco uso, entre quienes implementan esta información automatizada, para la toma de decisiones (28\%). 
Según el Informe Global de Tecnología de la Información del Foro Económico Mundial 2016, Finlandia, Suiza, Suecia, Israel, Singapur, los Países Bajos y los Estados Unidos tienen el liderazgo global en la generación de impacto económico a partir de las inversiones en Tecnologías de Información y Comunicación (TIC). Según se explica en World Economic Forum, basado en el Networked Readiness Index (NRI), este grupo de economías de alto rendimiento tiene un puntaje $33 \%$, en promedio, más alto que otras economías avanzadas y $100 \%$ más que las economías emergentes y en desarrollo. Estos países son conocidos por ser los primeros y más entusiastas de las TIC y su aparición es significativa, ya que demuestra que los beneficios son más amplios si la adopción de las TIC -junto con un entorno que facilita una regulación sólida, una infraestructura de calidad y la adopción de capacidades, entre otros factores.

El Informe Global de Tecnología 2016, informa que Colombia mantiene el mismo puntaje que el año 2015, pero desciende cuatro posiciones hasta el puesto 68 porque otros países mejoraron sus resultados. La adopción de TIC entre la población siguió aumentando a un ritmo acelerado: hubo 45,1 suscripciones de banda ancha móvil por cada 100 personas en 2014, frente a 25,0 en 2013 y 3,7 en 2011. Este aumento en el uso individual no ha sido acompañado por una tendencia similar entre empresas o dentro del gobierno. El alcance del uso de las TIC para operaciones B2B y B2C, así como para la creación de nuevos modelos comerciales, se ha estancado en los últimos años.

El ambiente político y comercial general en el país sigue siendo su principal debilidad, con

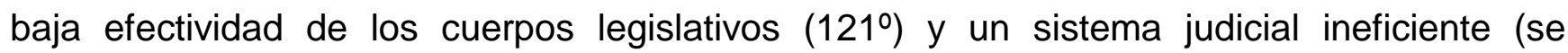
requieren 1,288 días para hacer cumplir un contrato, ocupando el 133ำ lugar en el mundo en este indicador). La tributación también sigue siendo desproporcionadamente alta, a una tasa del 69,7 por ciento (la sexta más alta entre los países de la muestra).

Esta situación revela los principales obstáculos para alcanzar el nivel de los países que hacen parte de la Organización para la Cooperación y el Desarrollo Económico, Ocde. El 
Ministerio de Comunicaciones de Colombia presiona a las empresas que prestan servicio de telecomunicaciones para que mejoren la velocidad mínima de banda ancha antes de 2019. El Plan Nacional de Desarrollo 2014-2018 estableció esta obligación como una manera de aumentar el nivel de conectividad establecido por el Gobierno Nacional, el cual aspira a llegar a 27 millones de conexiones de banda ancha en diciembre de 2018 (Enter.co, 2017, p. 27)

\section{Conclusiones}

Se refleja un excelente avance en la profesionalización en América Latina, el acceso a niveles de posgrado ha contribuido con este avance en Colombia, particularmente.

El uso de Big Data es una tendencia en proceso de apropiación por parte de los comunicadores y relacionistas colombianos. Están en sintonía con el desarrollo particular en la región, y con los retos que se plantean para todos los profesionales que participaron en la edición 2016-2017 del Latinamerican Communication Monitor.

Los mayores retos para sintonizarse con el aprovechamiento de Big Data en las estrategias de comunicación y de relaciones públicas, tienen que ver con la habilitación en análisis y procesamiento de los datos, con necesidad de adquirir capacidades técnicas, además de capacidad analítica. La tendencia del Big Data deja a los profesionales colombianos grandes desafíos en cuanto a adquirir más habilidades analíticas y técnicas.

Tradicionalmente se veía la función de divulgación como la mayor tarea en las organizaciones; esto ha cambiado y exige a los profesionales ser más analíticos y menos operativos, más coordinados sobre la interpretación y proyección de los datos que proveen los diferentes departamentos, de manera integrada, para dar su punto de vista y orientar estratégicamente, con previsión, con proyección y con conocimiento del entorno social, económico y, por supuesto, comunicacional. 
La destinación de tiempo laboral para las actividades operativas y de gestión comunicacional, limitan la ocupación en actividades de orientación, alineación y, por supuesto, de análisis y aprovechamiento de datos para generar conocimiento nuevo sobre las relaciones y comunicaciones estratégicas de organizaciones y clientes.

La actualización y participación en el debate acerca de Big Data, se convierte en una prioridad para los comunicadores y relacionistas colombianos, toda vez que en el país se desarrolla una política pública para tratamiento y uso de datos, tendiente a la optimización de información para mejoramiento del Estado democrático. La ausencia en este debate significará una brecha en la orientación ética y democrática del uso de información sobre las interacciones de los ciudadanos en el mundo digital.

Las comparaciones entre regiones permiten identificar percepciones diferentes sobre un mismo fenómeno, por esto, la influencia cultural es un aspecto a analizar en futuros artículos.

\section{Bibliografía}

Cukier, K. (2017) Big data, qué es y por qué es tan importante. Revista Vanguardia Dossier No.63, pp 70-73. Barcelona.

Castells, M. El panóptico digital. Revista Vanguardia Dossier No.63, pp 74-77. Barcelona.

Enter.co. (2016) El gobierno se quiere montar en el bus del 'Big Data'. Revista Enter.co, No.205. pp 56-57. Bogotá.

Gandomi, A. \& Haider, M. (2015). Beyond the hype: Big data concepts, methods, and analytics. International Journal of Information Management, 35(2), 137-144.

Baller, S, Dutta, S, Lanvin, B. (2016) The Global Information Technology Report 2016. World Economic Forum. Geneva. Recuperado de http://reports.weforum.org/globalinformation-technology-report2016/?doing wp cron=1542836370.0622489452362060546875 Fecha de acceso: 21 de noviembre de 2018

Enter.co (2017) El gran salto de Colombia en banda ancha. Revista Enter.co, No.218. p.27. Bogotá. 
Hallahan, K., Holtzhausen, D., van Ruler, B., Verčič, D. \& Sriramesh, K. (2007). Defining strategic communication. International Journal of Strategic Communication, 1(1), 3-35.

Molleda, J. C., Moreno, Á., Athaydes, A., \& Suárez, A. M. (2012). Macroencuesta latinoamericana de comunicación y relaciones públicas. Revista Organicom, 7(13)

Moreno, A. Molleda, J., Suárez, A.M., Athaydes, A., Álvarez, A., Herrera, M. (2017). Latin American Communication Monitor LCM 2016-2017. Euprera. www.latincommunicationmonitor.com

Departamento de Planeación Nacional de Colombia. Recuperado de www.dpn.gov.co. Consultado en diciembre de 2017.

Informe Global de Tecnología de la Información del Foro Económico Mundial 2016. Global Information Technology Report 2016. Colombia. Recuperado de: http://reports.weforum.org/global-information-technology-report-

2016/economies/\#indexld=NRI\&economy=COL Consultado el 21 de noviembre de 2017. Traducción propia.

P\&M. (2018) Briefing de Agencias. Creatividad en tiempos de Big Data. La propuesta de valor de los servicios creativos. Revista Publicidad \& Mercadeo, No. 451 pp.38-40. Bogotá.

Robles, J.M. (2009) Ciudadanía digital. Introducción al nuevo concepto de ciudadano digital. Barcelona: Editorial UOC.

UNCTAD (2017) Informe sobre la Economía de la Información: Digitalización, Comercio y Desarrollo. Conferencia de las Naciones Unidas para el Comercio y Desarrollo. Suiza: Naciones Unidas. World Economic Forum, Recuperado de: https://www.weforum.org/reports/the-global-information-technology-report-2016).

\section{Forma de citar este artículo en bibliografías}

SUÁREZ MONSALVE, A.M. y ÁLVAREZ NOBELL, A. (2018): "Los desafíos de Big Data para los comunicadores y relacionistas colombianos, según datos del LCM 2016-2017", en Revista PANGEA № 9, páginas 1 a 21. Tenerife: Red Académica Iberoamericana de Comunicación. Recuperado el _ de _ de $2 \_$de: http://www.revistapangea.org 